\title{
A Novel Approach for Extraction of Features from LP Residual in Time-Domain for Speaker Recognition
}

\author{
G. Chenchamma \\ Department Of Ece,Limat \\ Madalavari Gudam, Vijayawada Rural, Ap, \\ India.
}

\author{
A.Govardhan \\ Prof And Hod Of Cse \\ Jntu, Kukatpally, Hyd, India
}

\begin{abstract}
The objective is to model the dominating speakerspecific source in the time-domain at different levels, namely, Subsegmental, segmental and supra-segmental. The speaker-specific source information contained in the LP residual. Hence, LP residual contains different speaker-specific information at different levels. At each level features are extracted using proposed method called Hidden Markov models (HMM) and it is compared with existing Gaussian Mixture model (GMM). The experimental results demonstrates that the performance of Subsegmental level is more than the other two levels. However, the evidences from all the three levels of processing seem to be different and combine well to provide improved performance than the state-of -art speaker recognition system and demonstrating different speaker information captured at each level of processing. Finally, the combined evidence from all the three levels of processing together with vocal tract information further improves the speaker recognition performance. Experiments were conducted on TIMIT database using Gaussian Mixture Models (GMM's) and Hidden Markov models (HMM's). Comparing both results the proposed model HMM is better than the existing model GMM.
\end{abstract}

\section{Keywords}

Subsegmental, segmental, suprasegmental, LP residual, Hidden Markov models (HMM).

\section{INTRODUCTION}

The speaker information in the speech signal is attributed to the physiological and behavioral aspects of a person [1]. The physiological aspects are due to the vocal tract and excitation source that involved in the speech production $[1,2]$. The behavioral aspects involves factors like speaking rate accent etc.,[2]. The shape, size and the dynamics associated with the vocal tract contribute to the speaker characteristics. On the similar lines, the shape, size and the dynamics associated with vocal folds contribute to the speaker characteristics. State of the art speaker recognition system mostly use vocal tract related speaker information represented by the spectral or Cepstral features like linear prediction Cepstral coefficients LPCC or MFCC [6, 8, 9] these features provides good recognition performance. The reason may be that, they nearly represent complete vocal tract information. However, under degraded conditions the spectral or Cepstral features give poor performance. Hence there is a need for deriving robust features for SR. The speech production and perception theory indicate that the source contains speaker information and also more robust due to its impulsive nature. Motivated us to explore methods for modeling the speaker-specific source information from the complete source $[2,17,18$, $20,25,26,29,31]$. These attempts demonstrate that the source indeed significant speaker information .However, the recognition performance is far better than the vocal tract information. The reason may be that the methods employed in representing source information may not model all aspects of speaker information. By that we mean, LPCC or MFCC captures formants and their bandwidth information characterizing the vocal tract completely, pitch is only one aspect of speaker information due to source. Thus to improve the performance of source features, methods need to developed that tries to capture the complete source information. Source information contained in the LP residual of the speech signal [22 \& 23]. The LP residual can be processed in time, frequency, Cepstral or time frequency domains to extract and model information. $[9,22,29]$. Processing of LP residual in time-domain has the advantage that the artifacts of digital signal processing like block processing or windowing effect that creep in other domains of processing like will be negligible. Thus processing LP residual in time-domain is expected to model the speaker information in the best possible manner. Much work is not done on processing of LP residual at different levels. A unified frame work may be evolved where a given LP residual is processed at sub-segmental, segmental and supra-segmental levels. Extract features at each level and model the speaker information using Gaussian Mixture Models.

The rest of the paper is organized is as follows: Section 2 describes the proposed sub-segmental, segmental and supra-segmental analysis of LP residual approach for modeling speaker information from the LP residual .Section 3 results and discussions. The last section summarizes the present work with a mention on the scope for future work. 


\section{PROCESSING OF LP RESIDUAL IN TIME-DOMAIN}

In LP residual of speech production, each sample of speech is predicted as a linear combination of the past ' $p$ ' samples, where $p$ represents the order of prediction [12]. If $s(n)$ is the present sample, then it is predicted by the past $\mathrm{p}$ samples as

$$
\hat{\mathrm{S}}(\mathrm{n})=-\sum_{k=1}^{p} a_{k} s(n-k)
$$

Where, $\mathrm{a}_{\mathrm{k}} \mathrm{s}$ are the LP coefficients (LPCs) computed by minimizing the mean square prediction error. The error between the actual and the predicted sample value is called as the prediction error or LP residual and is given by

$$
\begin{aligned}
& \mathrm{r}(\mathrm{n})=\mathrm{s}(\mathrm{n})+\sum_{k=1}^{p} a_{k} s(n-k) \text {---- (2) } \\
& \mathrm{A}(\mathrm{z})=1+\sum_{k=1}^{p} \mathrm{a}_{\mathrm{k}} \mathrm{z}^{-\mathrm{k}}-\cdots-- \text { (3) }
\end{aligned}
$$

The predicted samples $\hat{\mathrm{S}}(\mathrm{n})$ model the vocal tract information in terms of (LPCs) [2,3]. The suppression of this information from the speech signal $s(n)$ that results in the LP residual $\mathrm{r}(\mathrm{n})$ is therefore mostly contains source information .The representation of source information in the LP residual depends upon the order of prediction. In prasanna et al [18], it was shown that for a speech signal sampled at $8 \mathrm{kHz}$, the LP residual extracted using LP order in the range 8-20 best represents the speaker-specific source information. The example of speech signal and LP residual is shown in figs $1 \mathrm{a}$ and $1 \mathrm{~b}$.
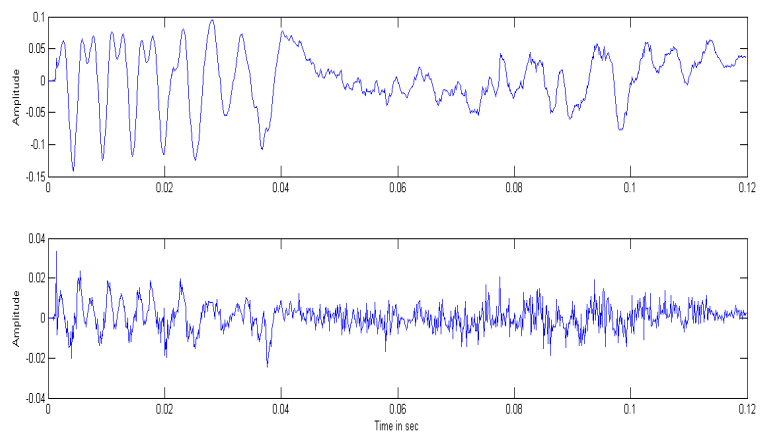

Fig. 1: a) Speech signal /aa/ b) Corresponding $12^{\text {th }}$ order LP residual

In this section, we describe the methods employed in processing the LP residual to extract speaker information. In extracting such information we consider subsegmental, segmental and suprasegmental level processing of LP residual. In subsegmental processing features are derived to represent the speaker information present mostly within one glottal cycle. In segmental processing, features are derived to represent the speaker information mostly related to pitch and energy of the excitation present across 2-3 glottal cycles. In suprasegmental level processing, features are derived to represent the prosodic aspects of the information present across about 25-50 glottal cycles.

\subsection{Speaker information from sub segmental processing of LP residual.}

At the subsegmental, speaker information present mostly within one glottal cycle is modeled. This information may be attributed to the activity like opening and closing glottal characteristics. To model this information, the LP residual is blocked into frames of $5 \mathrm{msec}$ with a shift of $2.5 \mathrm{msec}$. For $5 \mathrm{msec}$ at $8 \mathrm{kHz}$, they have 40 samples one such frame is shown in fig 2.1(a) and its spectrum is shown in fig 2.1(b). The largest amplitude of the samples of the vector indicates the strength of excitation. The samples in the vector represent the sequence information of glottal. Since these frames are obtained from the LP residual sampled at $8 \mathrm{kHz}$, they will have excitation source present as the fine variations represented by frequency components up to $4 \mathrm{kHz}$. These frames of LP residual samples in the time-domain are used as the feature vectors to represent speaker information at the subsegmental level and used for developing speaker recognition experiments using GMM (Gaussian Mixture Models). The nature of the LP residual that will be processed at the sub segmental level is one shown in fig.2.1 (a), this is nothing but the original LP residual.
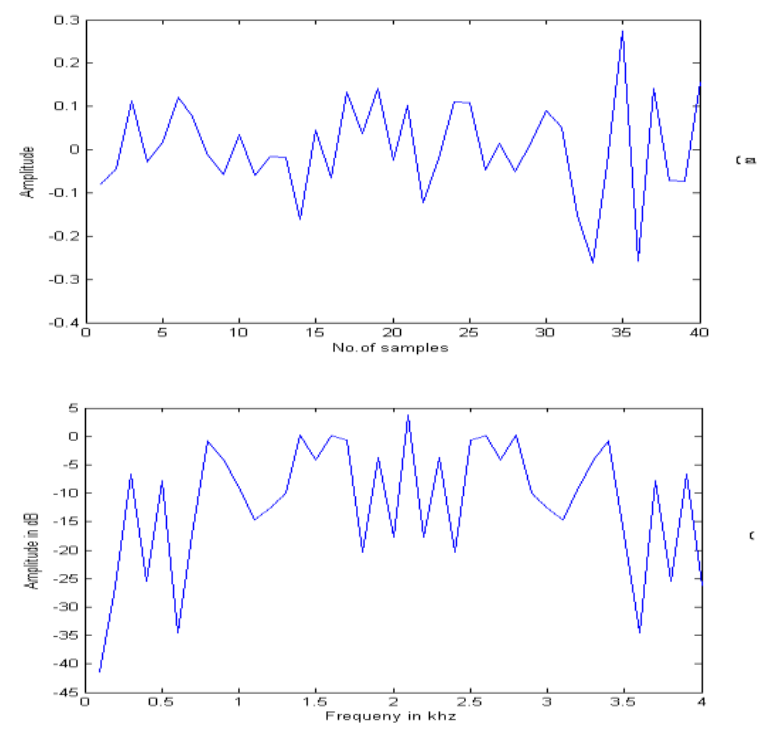

Fig 2.1: a) Subsegmental sequence b) Spectrum of Subsegmental of $L P$ residual.

The results of identification experiment are given in the Tables 1-2. In the tables the performance of the baseline system like vocal tract based features namely, MFCCs sampled at $8 \mathrm{kHz}$ with frame size $20 \mathrm{msec}$ and frame shift $10 \mathrm{msec}$ is given in the tables 12 . Hence the performance of Subsegmental level is more compared to the state-of-the-art speaker recognition system.

The results show that subsegmental features provide good performance and it contains dominant speaker information. However the performance is comparatively better than the vocal tract features. The better performance demonstrated that the subsegmental features contain dominant speaker - 
specific source information comparing with the remaining two levels.

\subsection{Speaker information from segmental processing of $L P$ residual}

At the segmental level, speaker information present in two to three glottal cycles is modeled. This information may be attributed mostly to pitch and energy. Speaker information represented by variations within a glottal cycle has already been modeled by sub segmental analysis. In segmental level processing of LP residual, other information that can be observed at the segmental level needs to be emphasized. For this we propose to decimate the LP residual by a factor 4 so that the sampling rate becomes $2 \mathrm{kHz}$ and we may have source information up to $1 \mathrm{kHz}$. The decimated LP residual is shown in Fig. 2(a). Even after decimation, the dominant speaker information at the segmental level, that is, pitch and energy information still can be preserved. Moreover, in segmental level processing, LP residual frames of 20 msec duration are used as the feature vectors. For 20 msec at $8 \mathrm{kHz}$, the feature vectors with 160 samples are of very large dimension for building the models. By decimating the LP residual by a factor 4 , the dimension of the feature vectors is reduced to 40 samples per vector which is equal to the Subsegmental feature vectors length. Since the LP residual is decimated by a factor 4 , we prefer to compute the feature vectors for every 2.5 msec frame shift so that the number of feature vectors will remain same as the subsegmental features. One such feature vector derived from the decimated LP residual is shown in Fig. 2(a). It contains mainly the pitch and energy information. The fine variations within the glottal cycle are suppressed by smoothing. Similar observation can also be made from the spectrum of the feature vector shown Fig. 2(b). The periodicity and the amplitude of the spectrum clearly represent the pitch and energy information. This observation indicates that segmental feature vectors reflect different aspect of source information compared to Subsegmental feature vectors. This will also be confirmed from the comparison study in Sect. 2.4. The effectiveness of these features is evaluated from the identification experiment. The results are given in the third column of the Table 1 and Table 2, respectively. The high performance shows that segmental features contain good speaker information, even better than those contained at the sub segmental level. This shows that the pitch and energy may be dominating speaker-specific source information. Further, the recognition performance is comparatively poor than vocal tract features. The same reason of incomplete representation of speaker information may be attributed. The segmental source features are relatively more robust compared to both vocal tract as well as sub segmental features and also requires fewer databases. The experiments conducted on TIMIT database.
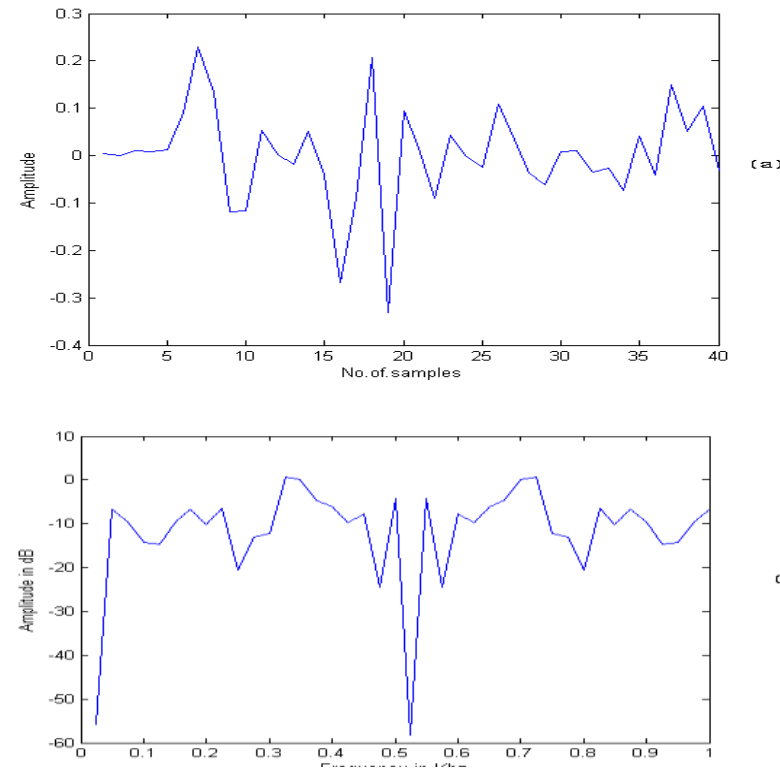

Fig 2.2: a) Segmental sequence b) Spectrum of segmental of LP residual

\subsection{Speaker information from suprasegmental processing of LP residual}

Subsegmental processing models speaker information up to $4 \mathrm{kHz}$. Segmental processing models speaker information up to $1 \mathrm{kHz}$. Beyond that $\mathrm{LP}$ residual also contains some speaker information at very low frequency range, that is, may be less than $100 \mathrm{~Hz}$. For example the variation in pitch and energy across several glottal cycles [2]. In capturing such information, we need to process the LP residual at the suprasegmental level, for example, with frames of 100-300 msec range. For the LP residual sampled at $8 \mathrm{kHz}$, the feature vectors from such frames will be of very large dimension for building models. We prefer to decimate the LP residual by a factor 50 so that the sampling rate becomes $160 \mathrm{~Hz}$ and we may have the source information up to $80 \mathrm{~Hz}$. The dimension of the feature vector is also reduced by 50 factors. Further, the high frequency information that is already modeled by subsegmental and segmental level processing will be smoothed out. Therefore in suprasegmental level processing of LP residual, we decimate the LP residual by a factor of 50 and process in frames of $250 \mathrm{msec}$ with shift of $6.25 \mathrm{msec}$. The frame size is decided so that the dimension of the feature vectors will remain same as in subsegmental and segmental processing. However, the minimum possible frame shift in this case is $6.25 \mathrm{msec}$ which corresponds to one same shift. Figure 2.3(b) shows a suprasegmental feature derived from the decimated residual shown in Fig. 2.1(a). The fast varying components of the original LP residual are eliminated and it mostly represents the long term variations. This can also be observed from the spectrum of the shown feature vector from Fig. 2.3(b). Information present in the smoothed spectrum is up to $80 \mathrm{~Hz}$. The periodicity and other high frequency related information are absent. 

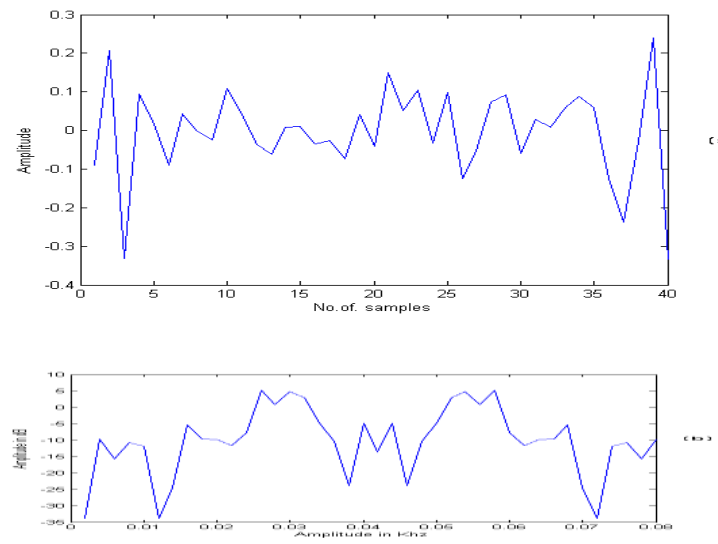

Fig 2.3: a) Suprasegmental sequence b) Spectrum of suprasegmental LP residual

The speaker information present in these features is verified from the recognition experiments as performed earlier. The results of the identification experiments are given in the fourth column of the Table 1 and Table 2, respectively. Results show that supra segmental level features contain some speaker information. Further, the recognition performance is significantly poor compared to sub segmental, segmental and vocal tract information. The poor result indicates that the supra segmental features may have large intra-speaker variability. The other major factor is text independent mode of operation. However, it may contain different aspect of speaker information (Long-term information) and hence may combine well with other features.

\subsection{Combining evidences from Sub segmental, segmental and supra- segmental levels of LP residual.}

By the way of deriving each feature, the information present at sub segmental, segmental and supra segmental levels are different and hence may reflect different aspect of speaker specific source information. By comparing their recognition performance it can be observed that the sub segmental features provide best performance. Thus the sub segmental features may have more speakerspecific evidence compared to other level features. The different performances in the recognition experiments indicate the different nature of speaker information present. In this section we use confusion patterns explain the different nature of the speaker information present in the proposed features and their usefulness for combined use in speaker recognition. In case of identification, the confusion pattern of features is considered as an indication of the different nature of information present. In the confusion pattern, principal diagonal represents correct identification and the rest represents miss classification. Figure 3 shows the confusion patterns of the identification results conducted for all the proposed features using TIMIT databases, respectively. In each case, the confusion pattern is entirely different. The decisions for both true and false identification are different. This indicates that they reflect different aspect of source information. This may help in combining the evidences to further improve the recognition performance from the complete source perspective.
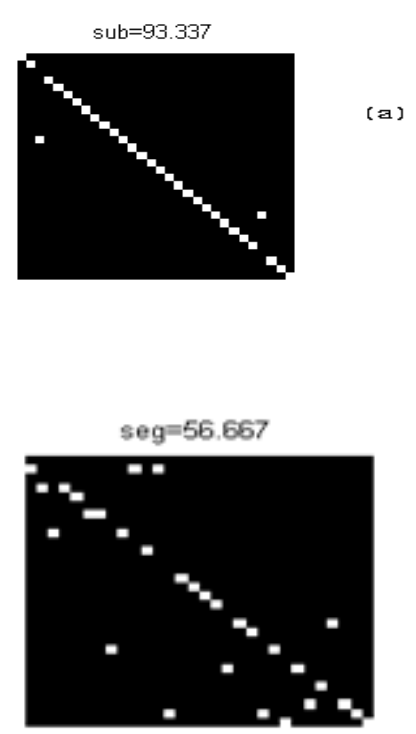

(b)

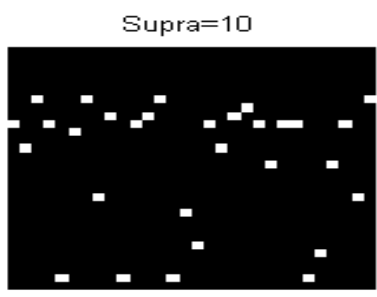

(c)

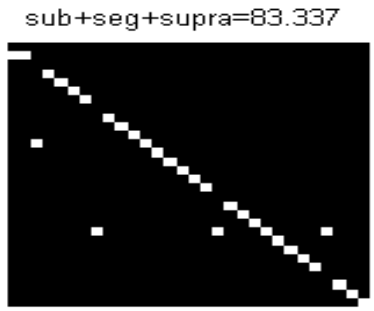

(c)

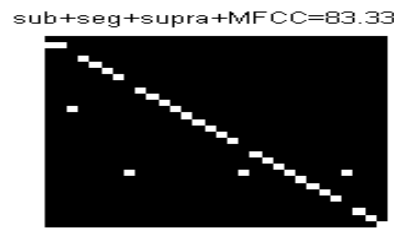

(e)

Fig 3: Confusion pattern of Sub, Seg, Supra, SRC $=$ sub+seg+supra and SRC+MFCC information for recognition of 38 speakers from TIMIT database. 


\section{RESULTS}

The role of dominant speaker-specific source characteristics (Variety of speaker-specific information), pitch and energy and long-term characteristics means low frequency information represented at subsegmental, segmental and suprasegmental levels, respectively, are examined for the task of SR for training data and testing data and number of mixtures. Results demonstrated that the performance of SR for variety of speaker characteristics represented at subsegmental level is more than the baseline model (MFCCs $6^{\text {th }}$ and $8^{\text {th }}$ column of Table 1), segmental and supra segmental models using GMM (Table 1). Combing evidences from each level improves performance of speaker recognition system is far better than the SR with vocal tract features and this is compared with the proposed model called ergodic HMM since it is more robust to model temporal characteristics of speech signal. This is the motivation to use HMM for distributing the features of LP residual at subsegmental, segmental and suprasegmental levels. Hence, the improved speaker recognition is shown in Table 2 at 2 states and Figs 5(a) and (b) in which performance is maximum at 2 states and observed that performance is reduced to increase states.
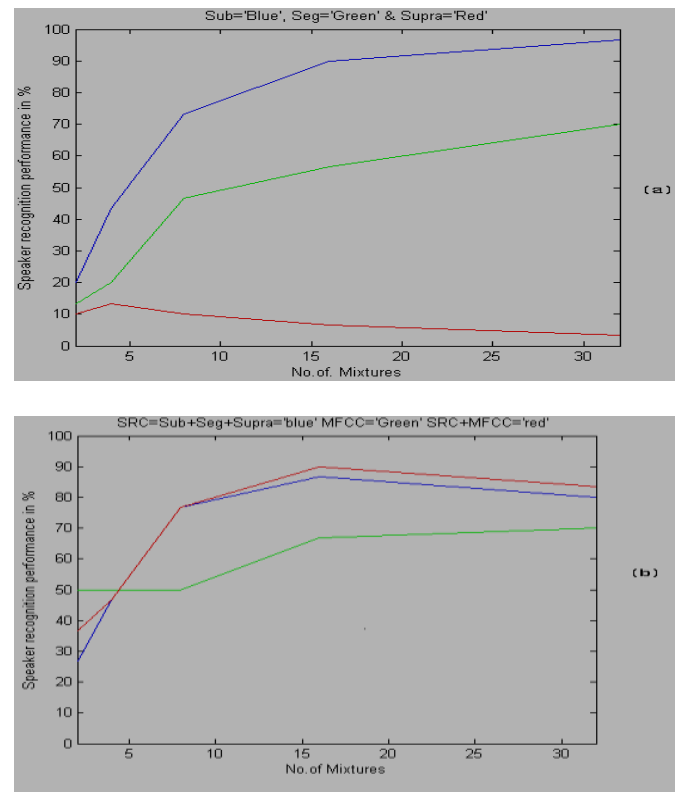

Fig. 4: The performance of speaker recognition system of Table 1 for a) Subsegmental(Sub) ,segmental (Seg) and suprasegmental (Supra) levels and b) Combined Sub+Seg+Supra and along with MFCC's.

\section{Summary and Conclusions:}

In this paper, we described a method for the extraction of complete source information by the time-domain analysis of the LP residual. Speaker specific information in the LP residual includes those within one glottal cycle. Pitch and energy across two to three cycles and variation of the pitch and energy across several glottal cycles. In the proposed methods, speaker information within one glottal cycle is extracted by the sub segmental processing of the LP residual. Pitch and energy contour information is extracted from the suprasegmental processing of the LP residual. To model the speaker information effectively using HMM compared to GMM. The segmental and suprasegmental level information is decimated by a factor of 4 and 50, respectively. Experimental results shows that subsegmental, segmental and suprasegmental levels contain speaker -specific source information. Further combining the evidences from each level, the performance improvement indicates the different nature of speaker information at each level. The performance of the speaker recognition is improved using HMM compared to the existing model GMM

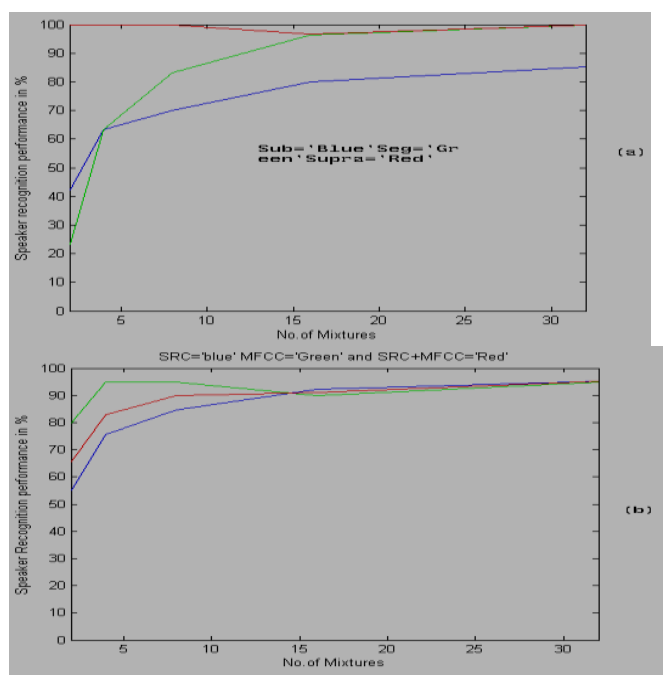

Fig.5: a). The performance of speaker recognition system for 2-state a) Subsegmental(Sub) ,segmental(Seg) and suprasegmental(Supra) levels and b) Combined Sub+seg+supra along with MFCC' 
Table 1: Subsegmental (Sub), segmental (Seg) and supra segmental of LP residual information for speaker recognition using Gaussian Mixture Model(GMM) of 38 speakers from TIMIT database. Each speaker spoken 10 sentences, among them 6 used for training 4 used for testing per speaker.

\begin{tabular}{|l|l|l|l|l|l|l|}
\hline $\begin{array}{l}\text { No .of } \\
\text { Mixtures }\end{array}$ & Sub & Seg & Supra & $\begin{array}{l}\text { SRC= } \\
\text { Sub+seg+supr } \\
\text { a }\end{array}$ & MFCC & $\begin{array}{l}\text { Sub+Seg+Supr } \\
\text { a+MFCC }\end{array}$ \\
\hline 2 & 20 & 13.3333 & 10 & 26.667 & 50 & 36.667 \\
\hline 4 & 43.3333 & 20 & 13.3333 & 46.667 & 50 & 46.667 \\
\hline 8 & 73.3333 & 46.6667 & 10 & 76.667 & 50 & 76.667 \\
\hline 16 & 90 & 56.667 & 6.6667 & 86.6667 & 66.6667 & 90 \\
\hline 32 & 96.667 & 70 & 3.3333 & 80 & 70 & 83.337 \\
\hline
\end{tabular}

Table 2: 2 Subsegmental (Sub), segmental (Seg) and supra segmental of LP residual information for speaker recognition using hidden Markov Models(HMM) of 38 speakers from TIMIT database. Each speaker spoken 10 sentences, among them 6 used as training 4 used as testing per speaker.

\begin{tabular}{|l|l|l|l|l|l|l|}
\hline $\begin{array}{l}\text { No. of } \\
\text { mixtures }\end{array}$ & Sub & Seg & Supra & SRC = Sub+seg+supra & MFCC's & SRC+MFCC's \\
\hline 2 & 42.22 & 23.3333 & 100 & 55.18 & 80 & 65.5 \\
\hline 4 & 63.33 & 63.3333 & 100 & 75.5555 & 95 & 83 \\
\hline 8 & 70 & 83.3333 & 100 & 84.6667 & 95 & 89.8888 \\
\hline 16 & 80 & 96.3333 & 96.6667 & 92.1111 & 90 & 91.1111 \\
\hline 32 & 85.33 & 100 & 100 & 95.1111 & 95 & 95.0556 \\
\hline
\end{tabular}

\section{REFERENCES}

[1] Ananthapadmanabha,T. V., \& Yegnanarayana, B. (1979). Epoch extraction from linear prediction residual for identification of closed glottis interval. IEEE Transactions on Acoustics, Speech, and Signal Processing, ASSP-27, 309-319.

[2] Atal, B. S.(1972). Automatic speaker recognition based on pitch contours. The Journal of the Acoustical Society of America, 52(6), 1687-1697.

[3] Atal, B. S.(1974). Effectiveness of linear prediction characteristics of the speech wave for automatic speaker identification and verification. The Journal of the Acoustical Society of America, 55(6), 13041312.

[4] Cohen, L. (1995). Time-frequency analysis: theory and application. Signal processing series. Englewood Cliffs: Prentice Hall.

[5] Davis, S. B., \& Mermelstein, P. (1980). Comparison of parametric representations for monosyllabic word recognition in continuously spoken sentences. IEEE Transactions on Acoustics, Speech, and Signal Processing, 28(28), 357-366.

[6] Ezzaidi, H., \& Rouat, J. (2004). Pitch and MFCC dependent GMM models for speaker identification systems. In IEEE int. conf. on electrical and computer eng.: Vol. 1.

[7] Furui, S. (1981). Cepstral analysis technique for automatic speaker verification. IEEE Transactions on Acoustics, Speech, and Signal Processing, 29(2), 254-272.

[8] Hayakawa, S., Takeda, K., \& Itakura, F. (1997). Speaker identification using harmonic structure of 1p-residual spectrum. In Lecture notes in computer science: Vol. 1206. Audioand video-based biometric personal authentification (pp. 253-260). Berlin: Springer.

[9] Huang, W., Chao, J., \& Zhang, Y. (2008). Combination of pitch and MFCC, GMM super vectors for speaker verification. In IEEE int. conf. on audio, language and image process (ICALIP) (pp. 1335-1339).

[10] Jankowski, C., Kalyanswamy, A., Basson, S., \& Spitz, J. (1990). NTIMIT: A phonetically balanced, continuous speech, telephone bandwidth speech database. In Int. conf. on acoust. speech and signal process. (ICASSP), Albuquerque, NM (pp. 109112).

[11] Makhoul, J. (1975). Linear prediction: A tutorial review. Proceedings of the IEEE, 63(4), 561-580.

[12] Martin, A., Doddington, G., Kamm, T., Ordowski, M., \& Przybocki, M. (1997). The DET curve in assessment of detection task performance. In Proc. Eur. conf. on speech communication technology, Rhodes, Greece, Vol. 4 (pp. 1895-1898).

[13] Mary, L., \& Yegnanarayana, B. (2008). Extraction and representation of prosodic features for language and speaker recognition. Speech Communication, 50, 782-796.

[14] Mashao, D. J.,\& Skosan, M. (2006). Combining classifier decisions for robust speaker identification. Pattern Recognition, 39, 147-155.

[15] Murthy, K. S. R., \& Yegnanarayana, B. (2008). Epoch extraction from speech signal. IEEE Transactions on Speech and Audio Processing, 16(8), 1602-1613. 
[16] Murthy, K. S. R., \& Yegnanarayana, B. (2009). Characterization of glottal activity from speech signal. IEEE Signal Processing Letters, 16(6), 469472.

[17] Murthy, K. S. R., \& Yegnanarayana, B. (2006). Combining evidence from residual phase and MFCC features for speaker recognition. IEEE Signal Processing letters, 13(1), 52-55.

[18] Murthy, K. S. R., Prasanna, S. R. M., \& Yegnanarayana, B. (2004). Speaker specific information from residual phase. In Int. conf. on signal proces. and comm. (SPCOM).

[19] Nist speaker recognition evaluation plan (2003). In: Proc. NIST speaker recognition workshop, College Park, MD.

[20] Prasanna, S. R. M., Gupta, C. S., \& Yegnanarayana, B. (2006). Extraction of speaker-specific excitation information from linear prediction residual of speech. Speech Communication, 48, 1243-1261.

[21] Przybocky, M., \& Martin, A. (2000). The NIST1999 speaker recognition evaluation- an overview. Digital Signal Processing, 10, 1-18.

[22] Reynolds, D. A. (1994). Experimental evaluation of features for robust speaker identification. IEEE Transactions on Speech and Audio Processing, 2(4), 639-643.

[23] Reynolds, D. A. (1995). Speaker identification and verification using Gaussian mixture speaker models. Speech Communication, 17, 91-108.

[24] Reynolds, D. A., \& Rose, R. C. (1995). Robust textindependent speaker identification using Gaussian mixture speaker models. IEEE Transactions on Speech and Audio Processing, 3(1), 4-17.

[25] Sonmez, K., Shriberg, E., Heck, L., \& Weintraub, M. (1998). Modeling dynamic prosodic variation for speaker verification. In Proc. ICSLP' 98: Vol. 7 (pp. 3189-3192).

[26] Thevenaz, P., \& Hugli, H. (1995). Usefulness of the LPC-residue in text-independent speaker verification. Speech Communication, 17, 145-157.

[27] Wolf, J. J. (1972). Efficient acoustic parameters for speaker recognition. The Journal of the Acoustical Society of America, 51(2), 2044-2055.

[28] Yegnanarayana, B., Reddy, K. S., \& Kishore, S. P. (2001). Source and system feature for speaker recognition using AANN models. In Proc. IEEE int. con.acoust. speech and signal processing, Salt Lake City, UT, USA, May 2001 (pp. 409-412).

[29] Yegnanarayana, B., Prasanna, S. R. M., Zachariah, J. M., \& Gupta, C. S. (2005). Combining evidences from source, suprasegmental and spectral features for fixed-text speaker verification study. IEEE Transactions on Speech and Audio Processing, 13(4), 575-582.

[30] Zheng, N., Lee, T., \& Ching, P. C. (2007). Integration of complimentary acoustic features for speaker recognition. IEEE Signal Processing Letters, 14(3), 181-184.

[31] Zue, V., Seneff, S., \& Glassa, J. (1990). Speech database development at MIT: Timit and beyond. Speech Communication, 9(4), 351-356. 